\title{
Review Article \\ Thymic Regulatory T Cell Development: Role of Signalling Pathways and Transcription Factors
}

\author{
Mark Engel, ${ }^{1}$ Tom Sidwell, ${ }^{2}$ Ajithkumar Vasanthakumar, \\ George Grigoriadis, ${ }^{1,3}$ and Ashish Banerjee ${ }^{1}$ \\ ${ }^{1}$ Centre for Inflammatory Diseases, Monash Medical Centre, Southern Clinical School, Monash University, \\ Clayton, VIC 3168, Australia \\ ${ }^{2}$ Molecular Immunology Division, Walter and Eliza Hall Institute of Medical Research, Melbourne, VIC 3052, Australia \\ ${ }^{3}$ Department of Clinical Haematology, Central Clinical School, Melbourne, VIC 3000, Australia
}

Correspondence should be addressed to George Grigoriadis; george.grigoriadis@monash.edu and Ashish Banerjee; ashish.banerjee@monash.edu

Received 14 June 2013; Accepted 21 August 2013

Academic Editor: E. Shevach

Copyright ( $) 2013$ Mark Engel et al. This is an open access article distributed under the Creative Commons Attribution License, which permits unrestricted use, distribution, and reproduction in any medium, provided the original work is properly cited.

Regulatory T cells (Tregs) are a subset of CD4 T cells that are key mediators of immune tolerance. Most Tregs develop in the thymus. In this review we summarise recent findings on the role of diverse signalling pathways and downstream transcription factors in thymic Treg development.

\section{Regulatory T Cells: Where Do They Come from and Why Do We Need Them?}

The primary function of the mature $\mathrm{T}$ cell population is to mediate immune responses against a diverse array of foreign antigens, while remaining unresponsive to self-antigens. While the diversity of the T cell population is generated by the semirandom rearrangement of T cell receptor ( $\alpha \beta \mathrm{TCR})$ genes during development in the thymus, tolerance towards self is enforced both in the thymus and periphery known as central and peripheral tolerance, respectively. Fine-tuning the TCR repertoire of the T cell pool during intrathymic development is achieved via two ways: the delivery of survival signals to those cells that successfully ligate their TCRs to MHC molecules loaded with self-peptides with low to moderate affinity (positive selection) and the induction of apoptosis in those that recognise the same ligands strongly enough to be potential mediators of autoimmune disease (negative selection). The latter is aided by the transcription factor Aire (autoimmune regulator) which facilitates the thymic "promiscuous expression" of a diverse array of tissue specific antigens. The remaining cells that fail to recognise self-peptide MHC complexes, such as those which fail to express a mature $\alpha \beta \mathrm{TCR}$, undergo time-dependent apoptosis, termed "death by neglect" [1-3]. The ideal outcome of this stringent and inefficient process of thymic education is a mature T cell repertoire whose TCRs successfully bind selfMHC molecules, while remaining unresponsive to autoantigens. Unfortunately, "central tolerance" to self is incomplete even in healthy mice and humans, in which peripheral $\mathrm{T}$ cells with potentially autoreactive TCRs can be found. These cells are kept unresponsive by immune cells whose function is to execute "peripheral" tolerance, with the best studied population being the Foxp $3^{+} \mathrm{CD} 4^{+}$regulatory $\mathrm{T}$ (Treg) cells $[4,5]$.

Treg cells were first identified as $\mathrm{T}$ cells that express high levels of the surface molecule CD25 and are capable of suppressing autoimmune reactions $[4,6]$. Subsequent identification of various surface markers such as the glucocorticoid-induced TNF receptor (GITR), the cytotoxic T lymphocyte antigen 4 (CTLA4), and notably the forkhead box transcription factor Foxp3, which is a Treg-lineage specific transcription factor in mice, allowed further characterisation of these cells [7-9]. Most Tregs develop in the thymus (tTregs) [10], with "induced" or "adaptive" Tregs ( $p$ Tregs for peripherally derived Tregs) generated via diverse 


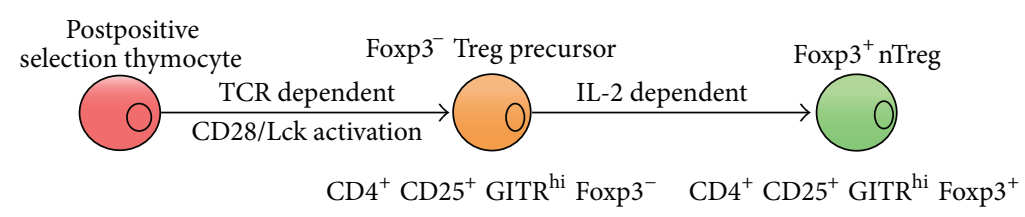

Figure 1: The stages of tTreg development. Thymic Treg development is achieved in two stages. Initially, postpositive selected thymocytes undergo TCR and CD28 dependent maturation into a Foxp3 $3^{-}$tTreg precursor population. A subsequent IL-2 dependent step leads to the development of Foxp3 expressing mature tTregs.

mechanisms from naive CD4 T cells [11] also contributing to the peripheral Treg pool. How tTregs, which reach functional maturity within the thymus, are generated has been the subject of considerable scientific debate. Early work with double-transgenic mice expressing a monoclonal TCR and cognate neoautoantigen within the thymus showed a definite skewing of the mature thymic CD4SP population towards a CD25 ${ }^{+}$Foxp $^{+}$regulatory phenotype [12-14], in a TCR affinity-dependent manner [13]. These data and similar subsequent work were the basis of the prevailing hypothesis that developing thymocytes are directed down the tTreg lineage by high-affinity TCR interactions with thymic peptide: MHC complexes [15].

\section{Role of TCR, CD28, and IL-2R Signalling in Thymic Treg Development}

While there are a number of unreconciled observations in the literature, objective data regarding discrete developmental steps prior to the appearance of Foxp3-expressing mature tTreg cells have emerged. A recent report has identified at least two distinct phases in tTreg development, based upon differential dependence on TCR signals [16]. They identified a Foxp $3^{-}$population (tTreg precursors) and demonstrated that it was enriched for tTreg-specific TCRs-indicating prior selection on the basis of TCR-and required only common- $\gamma$ chain $\left(\gamma_{c}\right)$ cytokines (predominantly IL-2) to differentiate into mature, Foxp3-expressing tTreg cells. Based on these observations the "two-step" model of tTreg cell development was proposed. In the first step, thymocytes undergo TCR and coreceptor dependent selection that gives rise to the $\mathrm{CD} 4^{+} \mathrm{CD} 25^{+} \mathrm{GITR}^{\text {hi }}$ Foxp $3^{-}$tTreg precursors $[17$, 18]. A subsequent TCR-independent IL-2/IL-15 dependent step results in Foxp3 expression marking the differentiation of tTreg precursors into tTregs $[16,19]$. This two-step model is the currently prevailing framework through which thymic tTreg development is analysed (Figure 1).

TCR ligation eventually leads to activation of the transcription factors NF-AT and NF- $\kappa \mathrm{B}[20,21]$. The activation of NF-AT depends on its dephosphorylation by the $\mathrm{Ca}^{2+}$ dependent phosphatase Calcineurin [22]. Although, both NF-AT and Calcineurin pathways have been implicated in tTreg development, their precise role in this process is controversial [23-25]. The redundant function of different NF-AT isoforms expressed in $\mathrm{T}$ lineage cells has made it difficult to ascertain the role of this group of transcription factors in tTreg development. NF-AT is required for the induction of Foxp3 transcription in vitro $[26,27]$. However, thymic regulatory $\mathrm{T}$ cell development and function are relatively normal in NF-ATc1/NF-ATc4 double deficient mice [28], with the suggestion that NF-ATc2 may compensate for the lack of NF-ATc1 and NF-ATc4 in these animals. Notably, mice deficient for NF-ATc2 and NF-ATc3 also exhibit normal tTreg development [29]. A severe defect in CD4 SP development due to a lack of NF-AT and ERK activation pose significant challenges to the study of Treg development in CalcinuerinB1 deficient mice [30-32]. Therefore, based on current experimental evidence, the Calcineurin/NF-AT pathway appears to be largely redundant in Treg development in the thymus. The apparent inconsistency between the requirement of NF-AT for Foxp3 expression in vitro (discussed later) and redundant function in tTreg development is further complicated by the finding that Calcineurin is not required for constitutive nuclear localisation of NF-AT in Tregs [33].

A number of reports have described a role for $\mathrm{NF}-\kappa \mathrm{B}$ in tTreg development [23, 34-37]. TCR dependent activation of $\mathrm{NF}-\kappa \mathrm{B}$ is mediated by a scaffold protein CARMA1, which belongs to the family of membrane associated guanylate kinases (MAGUK) [38-40]. TCR ligation leads to the calcium dependent activation of $\mathrm{PKC} \theta$, which in turn phosphorylates CARMA1 resulting in the recruitment of Bcl10 and MALT1. The CARMA1/Bcl10/MALT1 trimer, also known as the CBM complex, acts as a signalling platform that recruits and activates the IKK (I $\kappa \mathrm{B}$ kinase) complex, ultimately resulting in NF- $\kappa \mathrm{B}$ activation [41-43]. The Rel/NF- $\kappa \mathrm{B}$ family of transcription factors is comprised of 5 members, NF- $\kappa \mathrm{B} 1$, NF- $\kappa$ B2, RelA, RelB, and c-Rel. Homo- and heterodimers of $\mathrm{Rel} / \mathrm{NF}-\kappa \mathrm{B}$ proteins exist as latent complexes bound to $\mathrm{I} \kappa \mathrm{B}$ (inhibitor of $\kappa \mathrm{B}$ ) proteins in the cytosol. Signals emanating from diverse cell surface receptors including the TCR result in IKK dependent phosphorylation of $\mathrm{I} \kappa \mathrm{B}$, targeting it for ubiquitin-dependent proteasome-mediated degradation, thereby allowing NF- $\kappa \mathrm{B}$ dimers to enter the nucleus and bind to decameric $\kappa \mathrm{B}$ sites on regulatory elements of various genes $[44,45]$. NF- $\kappa \mathrm{B}$ regulated gene expression impinges on diverse processes downstream of the TCR including T cell development, survival, and cytokine production [46]. Given such functional diversity displayed by NF- $\kappa$ B in CD 4 T cell development/activation, it is not surprising that it plays a role in tTreg development. Mice deficient in PKC $\theta[23,37]$ or CARMA1 [34, 35] or those that lack Bcl10 [37] exhibit significant reduction in tTregs. A conditional deletion of $I k k \beta$ in DP thymocytes also results in loss of tTregs [36]. tTreg development has also been studied in some individual $N F-\kappa B$ knockout mice. While tTreg numbers are relatively normal 
in $N F-\kappa B 1^{-1-}$ mice [18], the absence of RelA results in a 2fold reduction in thymic $\mathrm{CD} 4^{+} \mathrm{CD} 25^{+} \mathrm{Foxp}^{+}$cells [47]. Of all the NF- $\kappa$ B family members, $c-R e l$ is the most important player in tTreg development with a $\sim 6-10$ fold reduction in tTreg numbers in $\mathrm{C}-\mathrm{rel}^{-/-}$mice $[18,47-51]$. It is important to note that like CARMA1, c-Rel also plays a nonredundant cell intrinsic role in the development of tTreg precursors [18, $35,52]$. It is believed that both CARMA1 and c-Rel deficient Treg precursors have impaired IL-2R signaling $[53,54]$ and as a consequence fail to efficiently differentiate into Foxp $3^{+}$ tTregs in response to IL-2 [52].

Signalling downstream of CD28 synergises with TCR signals to promote optimal Treg development [55]. Although the role of CD28 signalling in peripheral homeostasis and survival of Tregs is well appreciated [56-58], the precise mechanism by which costimulatory signals regulate tTreg development is poorly understood. Interaction of B7 with CD28 is believed to strengthen contact between the antigen presenting cell and developing thymocyte thereby promoting survival of the latter via IL-2 production and upregulation of prosurvival $\mathrm{Bcl}_{\mathrm{XL}}$ [59-61]. Both $\mathrm{CD} 28$ and $\mathrm{B} 7$ knockout mice have significant reduction in thymic Treg numbers $[18,55,57$, 58]. An elegant study by Tai et al. described a PYAP motif (aa 187-190) in the cytoplasmic tail of CD28 that is required for tTreg development. Apart from binding the tyrosine kinase Lck, this motif is also required for IL-2 production. Although transgenic overexpression of a wild-type CD28 transgene significantly rescued tTreg numbers in $\mathrm{Cd} 28^{-/-}$ mice, a mutant CD28 transgene where both Prolines in the PYAP motif were substituted with Alanine failed to do so [55]. Recent work has demonstrated that CD28 is also required for the generation of $\mathrm{t}$ Treg precursors. Using mixed bone marrow chimeras, a cell intrinsic role of CD28/Lck in development of tTreg precursors was described [18]. The requirement of Lck in CD28 dependent tTreg development is not restricted to generation of tTreg precursors as Lck activation is also required for expression and stabilisation of Foxp3 message [62]. Interestingly, IL-2 deficiency in mice has little to no impact on tTreg numbers $[63,64]$. However, IL-2 plays an important role in the survival of mature Tregs $[63,64]$.

\section{Transcriptional Control of foxp 3 in Thymic Tregs}

Humans with a mutation in their foxp 3 gene suffer from a spontaneous inflammatory disease called IPEX [65-67] (Immune dysregulation Polyendocrinopathy Enteropathy X linked syndrome). Additionally, a naturally occurring Foxp3 mutation in mice (Scurfy) results in a severe T cell dependent multiorgan autoimmune and inflammatory disease $[68,69]$. These findings necessitated closer examination of how the expression of this crucial transcription factor is controlled.

The architecture of the Foxp3 promoter along with its downstream cis regulatory elements determines Treg-specific expression and maintenance of Foxp3. The promoter of Foxp3 is located $\sim 6.6 \mathrm{~kb}$ upstream of its translational start site and has weak transcriptional activity [70] which is enhanced by the binding of several transcription factors (TFs) to the cis regulatory elements located in the introns. Two evolutionarily conserved noncoding sequences (CNS), Cns1 and Cns2, located in the first intron between the two $5^{\prime}$ UTR exons act as enhancer elements [70]. Additionally, an enhancer element, Cns 3 , located in the third intron immediately after the first coding exon serves an indispensable role in the induction of Foxp3 expression during thymic Treg development [50]. The importance of these CNSs in Foxp3 expression and Treg development was demonstrated by systematic deletion of these cis acting elements in mice [50]. Cns1 was shown to be dispensable for tTreg development but required for TGF $\beta$ dependent conversion of naive $\mathrm{CD} 4^{+} \mathrm{T}$ cells into Tregs. $\mathrm{Cns}^{-1-}$ mice did not display major defects in thymic Treg development. However, Cns 2 deletion adversely affected the stability of Foxp3 expression. In contrast, deletion of Cns3 resulted in a significant reduction in thymic Treg numbers. TCR, CD28, and IL-2 dependent signals activate and recruit TFs to the promoter and Cns 2 and $C n s 3$ enhancer elements to initiate Foxp 3 transcription and maintain stable expression of Foxp3 in tTregs. These enhancer regions are also amenable to epigenetic modifications, which serve as an additional layer of transcriptional control imposed on Foxp 3 expression.

\section{Differential DNA Methylation Regulates foxp3 Transcription}

The Cns2 element encompasses a CpG island that is hypomethylated only in thymic Tregs [48]. TCR signals result in Cns2 demethylation rendering this element permissive for the binding of transcription factors induced downstream of TCR, CD28, and the IL-2R [70-73]. Pharmacological inhibition of DNA methylation resulted in Foxp3 induction in conventional $\mathrm{CD}^{+} \mathrm{T}$ cells [74] supporting the notion that methylation of Cns2 is prohibitive for Foxp3 expression. Conversely, reducing the expression of the DNA methyltransferase, Dnmtl, using shRNA mediated knockdown also resulted in Foxp3 expression, reinforcing the importance of Cns2 demethylation in thymic Treg development [70]. In addition to the induction of Foxp3 transcription, demethylation of Cns2 is also required for stable Foxp3 expression [50]. Although forced demethylation of Cns2 is known to initiate Foxp3 transcription, the exact TCR dependent mechanism(s) that demethylates Cns2 during tTreg development is unclear. It is important to appreciate that the exact role of Cns2 is hard to define in the absence of further knowledge about factors that bind this region. The lack of a phenotype in $\mathrm{Cns}^{-/-}$mice may mean that Foxp3 expression proceeds unimpeded because a negative regulatory region (Cns2) is no longer there. In other words, the function of Cns2 demethylating factors is made redundant in $\mathrm{Cns}^{-/-}$mice.

Like Cns2, the Foxp3 promoter region has interspersed CpG motifs the methylation status of which is critical for the initiation of transcription. The Foxp3 promoter region is completely methylated in naive CD4 T cells and partially methylated in Tregs [70, 74]. However, unlike Cns2, promoter demethylation increases as Tregs mature and migrate to the periphery [75, 76]. Like Cns2, demethylation of the promoter is permissive for the binding of several TFs to initiate 


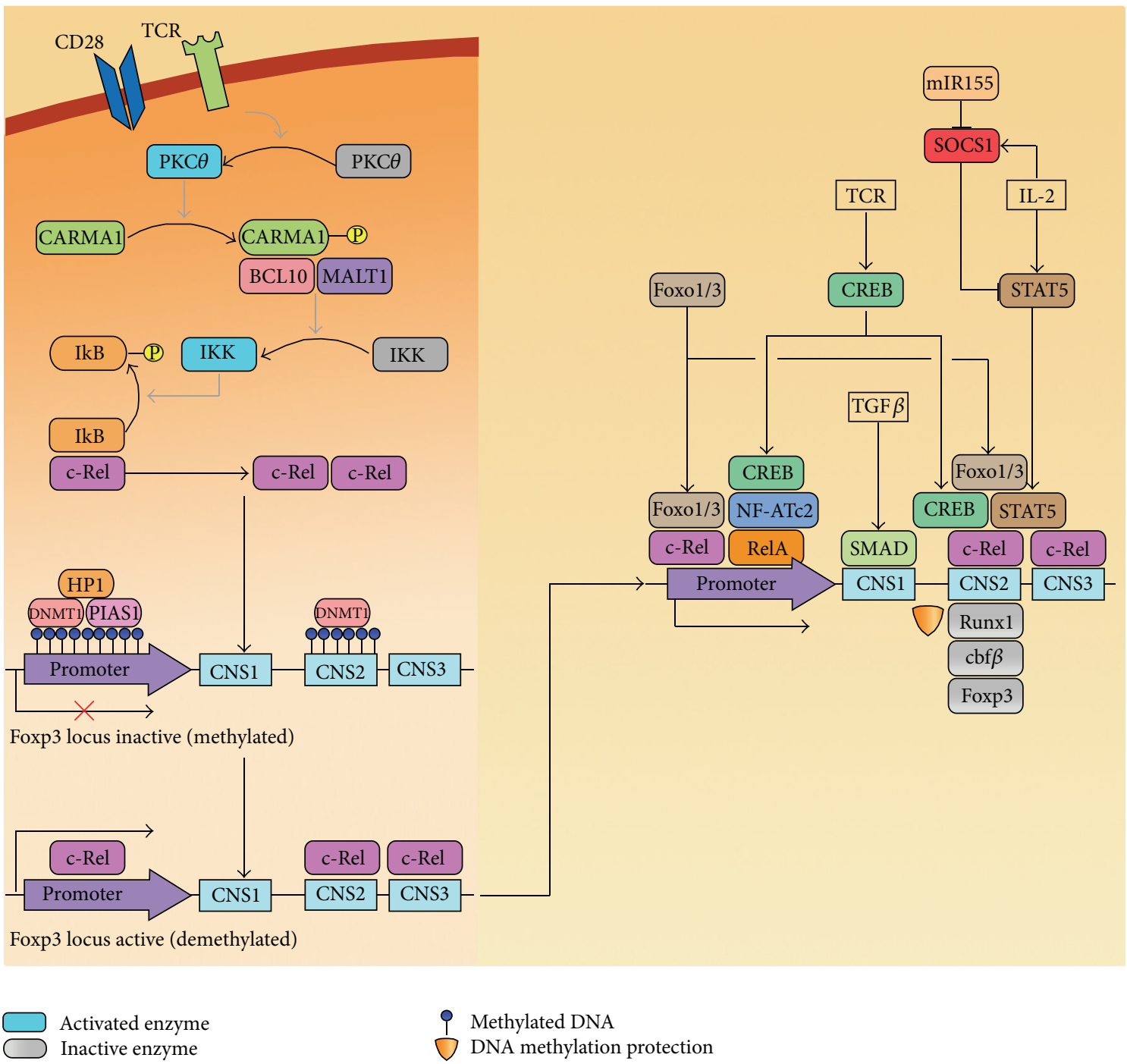

FIGURE 2: Transcription factors in tTreg development. Once engaged, cell surface receptors initiate c-Rel activation and nuclear entry. c-Rel binding to the Foxp3 promoter and Cns elements promotes epigenetic changes, including demethylation and chromatin remodelling (not pictured) of the Foxp3 locus. Several other transcription factors cooperate with c-Rel to initiate and preserve the stable expression of Foxp3 in tTregs.

the expression of Foxp3 in response to TCR, CD28, and IL-2R signals. Supporting this notion, a negative regulator of Treg development PIAS1 maintains the Foxp3 promoter in its methylated state by binding to it and recruiting DNA methyltransferases and Heterochromatin protein 1 resulting in the formation of a "repressive complex" [77]. This "repressive complex" collapses upon TCR signalling and the promoter becomes accessible to TFs to participate in the initiation of transcription [77].

\section{TCR- and IL-2-Induced Transcription Factors Induce Foxp3 Expression in tTregs}

Several TFs have been implicated as essential factors in Foxp3 expression. Primarily the NF- $\kappa$ B family member, c-Rel, has been demonstrated to be important in Foxp3 induction during tTreg development. The drastic reduction of tTreg numbers in $\mathrm{C}-\mathrm{rel}^{-/-}$mice is linked to a defect in the induction of Foxp3 expression [48]. To date, three different mechanisms have been proposed to explain the role of c-Rel in Foxp3 induction. Given TCR signalling is responsible for Cns2 demethylation with c-Rel being activated downstream of the TCR, it is possible that recruitment of c-Rel to Cns 2 results in its demethylation [48]. This hypothesis stems from the finding that c-Rel can bind to the methylated Cns 2 region in the Foxp3 locus [48]. Therefore, it is plausible that demethylation of the Cns2 region induced by the binding of c-Rel facilitates binding of other TFs resulting in the induction of Foxp3 transcription. Yet another study elegantly demonstrated that c-Rel nucleated an "enhanceosome" comprising other TFs 
including p65 (RelA), pCREB, and NF-ATc2 at the Foxp3 promoter [49]. The authors utilised in vitro differentiated Tregs (iTregs) to demonstrate that the recruitment of cRel and RelA to the Foxp3 promoter shortly after TCR stimulation led to the formation of this "enhanceosome." In the absence of c-Rel the enhanceosome failed to form suggesting the central role of c-Rel in this process. Finally, Zheng et al. demonstrated the in vitro binding of c-Rel to a CD28 response element located in the Cns3 region [50]. A similar reduction in tTregs observed in both $\mathrm{C}-\mathrm{rel}^{-/-}$and $\mathrm{Cns}^{-/-}$mice supports the model whereby c-Rel binding to Cns 3 results in thymic Foxp3 expression. Given Cns3 is permissive from the DP stage to tTregs, c-Rel can potentially access Cns 3 regardless of the Cns 2 methylation status. The binding of c-Rel to Cns 3 is also believed to induce chromatin remodelling at the adjacent $\mathrm{Cns} 2$ element. The resultant $\mathrm{Cns} 3$ c-Rel-induced permissive status of Cns2 can be utilised by other TFs for the initiation of Foxp 3 transcription.

The demethylated Cns2 region also recruits cyclicAMP response element binding protein (CREB)/activating transcription factor (ATF) in response to TCR signals [70]. Another group of proteins known to induce Foxp3 expression in tTregs belong to the Foxo family of TFs and include Foxol and Foxo3 that bind to the promoter and Cns2 elements to induce Foxp3 expression. [78]. TCR and CD28 stimulation results in activation and nuclear entry of c-Rel. In contrast, Foxo protein that are constitutively present in the nucleus are phosphorylated and inactivated by the PI3K/Akt signalling axis downstream of TCR. Therefore it is possible that Foxo proteins and c-Rel bind sequentially to the Foxp3 locus [78]. In addition to these transcription factors, IL-2-induced STAT5 also has a prominent role in controlling Foxp 3 expression [71-73]. IL-2 signalling induces increased levels of SOCS1, which negatively regulates STAT5 resulting in reduced Foxp3 expression. Therefore, efficient expression of Foxp 3 requires suppression of SOCS1 levels in Tregs. The microRNA mIR155 targets Socs 1 mRNA in Tregs and consequently maintains high levels of STAT5 expression [79]. Deletion of Mir155 in mice has been reported to result in upregulation of SOCS1 and subsequent inhibition of STAT5 mediated Foxp3 expression in tTregs [79, 80]. Sustained or stable expression of Foxp3 which is critical for tTreg-lineage stability is maintained by the TFs, Runxl, and $\operatorname{Cbf} \beta$. These TFs form a trimeric complex with Foxp3 and bind Cns2 establishing a feed forward loop ensuring stable Foxp3 expression [50]. Given these findings, we propose that c-Rel, known as the "pioneer TF," is the first TF to bind to the Foxp3 promoter, Cns3, and Cns2 following TCR ligation. The binding of c-Rel to Cns3 and methylated Cns2 results in demethylation of Cns2 facilitating subsequent recruitment of Foxol and Foxo3. Phospho-STAT5 induced by IL-2 follows soon after ultimately resulting in Foxp 3 transcription. It is important to note that once demethylated, the Cns $2 \mathrm{CpG}$ region is protected from methylating enzymes by the Foxp3-Runxl-Cbf $\beta$ complex [50]. In summary, we propose that the TCR-/IL2 -induced transcriptional activation of Foxp3 is initiated by c-Rel, which is then maintained by sequential binding of other TFs including Foxp3 itself (Figure 2).

\section{Concluding Remarks}

Since their discovery, regulatory $\mathrm{T}$ cells have been at the forefront of immunology research. Although we have made significant progress towards the understanding of their development and function a lot needs to be done to apply these findings in the clinic. Although a number of publications have reported modulation of Treg numbers and/or function in diverse disease, very little is known about the real impact of Treg modulation in disease onset and progression. It is critical to acknowledge that Tregs can be both beneficial and deleterious depending on the disease and stage. Therefore we believe that a careful analysis of Treg function in individual diseases is needed to meaningfully apply Treg based therapies in the clinic.

\section{Authors' Contribution}

Mark Engel, Tom Sidwell, and Ajithkumar Vasanthakumar contributed equally to the work.

\section{References}

[1] L. Klein, M. Hinterberger, G. Wirnsberger, and B. Kyewski, "Antigen presentation in the thymus for positive selection and central tolerance induction," Nature Reviews Immunology, vol. 9, no. 12, pp. 833-844, 2009.

[2] M. S. Anderson and M. A. Su, "Aire and T cell development," Current Opinion in Immunology, vol. 23, no. 2, pp. 198-206, 2011.

[3] A. D. Griesemer, E. C. Sorenson, and M. A. Hardy, "The role of the thymus in tolerance," Transplantation, vol. 90, no. 5, pp. 465-474, 2010.

[4] S. Sakaguchi, N. Sakaguchi, M. Asano, M. Itoh, and M. Toda, "Immunologic self-tolerance maintained by activated $\mathrm{T}$ cells expressing IL-2 receptor $\alpha$-chains (CD25): breakdown of a single mechanism of self-tolerance causes various autoimmune diseases," Journal of Immunology, vol. 155, no. 3, pp. 1151-1164, 1995.

[5] S. Sakaguchi, T. Yamaguchi, T. Nomura, and M. Ono, "Regulatory T cells and immune tolerance," Cell, vol. 133, no. 5, pp. 775-787, 2008.

[6] S. Sakaguchi, K. Fukuma, K. Kuribayashi, and T. Masuda, "Organ-specific autoimmune diseases induced in mice by elimination of T cell subset. I. Evidence for the active participiation of T cells in natural self-tolerance; deficit of a T cell subset as a possible cause of autoimmune disease," Journal of Experimental Medicine, vol. 161, no. 1, pp. 72-87, 1985.

[7] S. Hori, T. Nomura, and S. Sakaguchi, "Control of regulatory T cell development by the transcription factor Foxp3," Science, vol. 299, no. 5609, pp. 1057-1061, 2003.

[8] J. D. Fontenot, M. A. Gavin, and A. Y. Rudensky, "Foxp3 programs the development and function of CD4+CD25+ regulatory T cells," Nature Immunology, vol. 4, no. 4, pp. 330-336, 2003.

[9] R. Khattri, T. Cox, S. A. Yasayko, and F. Ramsdell, "An essential role for Scurfin in CD4+CD25+ T regulatory cells," Nature Immunology, vol. 4, no. 4, pp. 337-342, 2003.

[10] J. D. Fontenot, J. L. Dooley, A. G. Farr, and A. Y. Rudensky, "Developmental regulation of Foxp3 expression during ontogeny," Journal of Experimental Medicine, vol. 202, no. 7, pp. 901-906, 2005. 
[11] E. M. Shevach, T. S. Davidson, E. N. Huter, R. A. DiPaolo, and J. Andersson, "Role of TGF- $\beta$ in the induction of Foxp3 expression and T regulatory cell function," Journal of Clinical Immunology, vol. 28, no. 6, pp. 640-646, 2008.

[12] I. Apostolou, A. Sarukhan, L. Klein, and H. von Boehmer, "Origin of regulatory T cells with known specificity for antigen," Nature Immunology, vol. 3, no. 8, pp. 756-763, 2002.

[13] M. S. Jordan, A. Boesteanu, A. J. Reed et al., "Thymic selection of CD4+CD25+ regulatory $\mathrm{T}$ cells induced by an agonist selfpeptide," Nature Immunology, vol. 2, no. 4, pp. 301-306, 2001.

[14] M. A. Lerman, J. Larkin III, C. Cozzo, M. S. Jordan, and A. J. Caton, "CD4+ CD25+ regulatory T cell repertoire formation in response to varying expression of a neo-self-antigen," Journal of Immunology, vol. 173, no. 1, pp. 236-244, 2004.

[15] J. Cabarrocas, C. Cassan, F. Magnusson et al., "Foxp3+ CD25+ regulatory $\mathrm{T}$ cells specific for a neo-self-antigen develop at the double-positive thymic stage," Proceedings of the National Academy of Sciences of the United States of America, vol. 103, no. 22, pp. 8453-8458, 2006.

[16] C. W. J. Lio and C. S. Hsieh, "A two-step process for thymic regulatory T cell development," Immunity, vol. 28, no. 1, pp. 100111, 2008.

[17] C. W. J. Lio, L. F. Dodson, C. M. Deppong, C. S. Hsieh, and J. M. Green, "CD28 facilitates the generation of Foxp3-cytokine responsive regulatory T cell precursors," Journal of Immunology, vol. 184, no. 11, pp. 6007-6013, 2010.

[18] K. B. Vang, J. Yang, A. J. Pagán et al., "Linked T cell receptor and cytokine signaling govern the development of the regulatory $\mathrm{T}$ cell repertoir," Journal of Immunology, vol. 184, no. 8, pp. 40744077, 2010.

[19] M. A. Burchill, J. Yang, K. B. Vang et al., "Linked T Cell Receptor and Cytokine Signaling Govern the Development of the Regulatory T Cell Repertoire," Immunity, vol. 28, no. 1, pp. 112-121, 2008.

[20] S. Gerondakis, A. Banerjee, G. Grigoriadis et al., "NF- $\kappa$ B subunit specificity in hemopoiesis," Immunological Reviews, vol. 246, no. 1, pp. 272-285, 2012.

[21] F. Macian, "NFAT proteins: key regulators of T-cell development and function," Nature Reviews Immunology, vol. 5, no. 6, pp. 472-484, 2005.

[22] P. G. Hogan, L. Chen, J. Nardone, and A. Rao, "Transcriptional regulation by calcium, calcineurin, and NFAT," Genes and Development, vol. 17, no. 18, pp. 2205-2232, 2003.

[23] S. Gupta, S. Manicassamy, C. Vasu, A. Kumar, W. Shang, and Z. Sun, "Differential requirement of PKC- $\theta$ in the development and function of natural regulatory T cells," Molecular Immunology, vol. 46, no. 2, pp. 213-224, 2008.

[24] Y. Wu, M. Borde, V. Heissmeyer et al., "FOXP3 controls regulatory $\mathrm{T}$ cell function through cooperation with NFAT," Cell, vol. 126, no. 2, pp. 375-387, 2006.

[25] R. Zeiser, V. H. Nguyen, A. Beilhack et al., "Inhibition of $\mathrm{CD} 4+\mathrm{CD} 25+$ regulatory $\mathrm{T}$-cell function by calcineurindependent interleukin-2 production," Blood, vol. 108, no. 1, pp. 390-399, 2006.

[26] Y. Tone, K. Furuuchi, Y. Kojima, M. L. Tykocinski, M. I. Greene, and M. Tone, "Smad3 and NFAT cooperate to induce Foxp3 expression through its enhancer," Nature Immunology, vol. 9, no. 2, pp. 194-202, 2008.

[27] M. Vaeth, U. Schliesser, G. Muller et al., "Dependence on nuclear factor of activated T-cells (NFAT) levels discriminates conventional T cells from Foxp3+ regulatory T cells," Proceedings of the National Academy of Sciences of the United States of America, vol. 109, no. 40, pp. 16258-16263, 2012.

[28] M. Oh-hora and A. rao, "The calcium/NFAT pathway: role in development and function of regulatory T cells," Microbes and Infection, vol. 11, no. 5, pp. 612-619, 2009.

[29] T. Bopp, A. Palmetshofer, E. Serfling et al., "NFATc2 and NFATc3 transcription factors play a crucial role in suppression of CD4+ T lymphocytes by CD4+ CD25+ regulatory T cells," Journal of Experimental Medicine, vol. 201, no. 2, pp. 181-187, 2005.

[30] O. F. Bueno, E. B. Brandt, M. E. Rothenberg, and J. D. Molkentin, "Defective T cell development and function in calcineurin $\mathrm{A} \beta$ deficient mice," Proceedings of the National Academy of Sciences of the United States of America, vol. 99, no. 14, pp. 9398-9403, 2002.

[31] E. M. Gallo, M. M. Winslow, K. Canté-Barrett et al., "Calcineurin sets the bandwidth for discrimination of signals during thymocyte development," Nature, vol. 450, no. 7170, pp. 731-735, 2007.

[32] J. R. Neilson, M. M. Winslow, E. M. Hur, and G. R. Crabtree, "Calcineurin B1 is essential for positive but not negative selection during thymocyte development," Immunity, vol. 20, no. 3, pp. 255-266, 2004.

[33] Q. Li, A. Shakya, X. Guo et al., "Constitutive nuclear localization of NFAT in Foxp3+ regulatory T cells independent of calcineurin activity," Journal of Immunology, vol. 188, no. 9, pp. 4268-4277, 2012.

[34] B. D. Medoff, B. P. Sandall, A. Landry et al., "Differential requirement for CARMAl in agonist-selected T-cell development," European Journal of Immunology, vol. 39, no. 1, pp. 78-84, 2009.

[35] L. L. Molinero, J. Yang, T. Gajewski, C. Abraham, M. A. Farrar, and M. L. Alegre, "CARMA1 controls an early checkpoint in the thymic development of foxP3 + regulatory T cells," Journal of Immunology, vol. 182, no. 11, pp. 6736-6743, 2009.

[36] M. Schmidt-Supprian, G. Courtois, J. Tian et al., "Mature T cells depend on signaling through the IKK complex," Immunity, vol. 19, no. 3, pp. 377-389, 2003.

[37] M. Schmidt-Supprian, J. Tian, E. P. Grant et al., "Differential dependence of CD4+CD25+ regulatory and natural killer-like T cells on signals leading to NF- $\kappa \mathrm{B}$ activation," Proceedings of the National Academy of Sciences of the United States of America, vol. 101, no. 13, pp. 4566-4571, 2004.

[38] O. Gaide, B. Favier, D. F. Legler et al., "CARMAI is a critical lipid raft-associated regulator of TCR-induced NF- $\kappa$ B activation," Nature Immunology, vol. 3, no. 9, pp. 836-843, 2002.

[39] C. Jiang and X. Lin, "Regulation of NF-kappaB by the CARD proteins," Immunological Reviews, vol. 246, no. 1, pp. 141-153, 2012.

[40] D. Wang, Y. You, S. M. Case et al., "A requirement for CARMAI in TCR-induced NF- $\kappa$ B activation," Nature Immunology, vol. 3, no. 9, pp. 830-835, 2002.

[41] X. Lin and D. Wang, "The roles of CARMA1, Bcl10, and MALT1 in antigen receptor signaling," Seminars in Immunology, vol. 16, no. 6, pp. 429-435, 2004.

[42] K. Sommer, B. Guo, J. L. Pomerantz et al., "Phosphorylation of the CARMA1 linker controls NF- $\kappa \mathrm{B}$ activation," Immunity, vol. 23, no. 6, pp. 561-574, 2005.

[43] D. Wang, R. Matsumoto, Y. You et al., "CD3/CD28 costimulation-induced NF- $\kappa \mathrm{B}$ activation is mediated by recruitment of protein kinase $\mathrm{C}-\theta, \mathrm{Bcl} 10$, and $\mathrm{I} \kappa \mathrm{B}$ kinase $\beta$ to 
the immunological synapse through CARMAl," Molecular and Cellular Biology, vol. 24, no. 1, pp. 164-171, 2004.

[44] A. Hoffmann, G. Natoli, and G. Ghosh, "Transcriptional regulation via the NF- $\kappa \mathrm{B}$ signaling module," Oncogene, vol. 25, no. 51, pp. 6706-6716, 2006.

[45] C. Scheidereit, "I $\kappa \mathrm{B}$ kinase complexes: gateways to NF- $\kappa \mathrm{B}$ activation and transcription," Oncogene, vol. 25, no. 51, pp. 6685-6705, 2006.

[46] H. Oh and S. Ghosh, "NF- $\kappa$ B: roles and regulation in different CD4(+) T-cell subsets," Immunological Reviews, vol. 252, no. 1, pp. 41-51, 2013.

[47] I. Isomura, S. Palmer, R. J. Grumont et al., "c-Rel is required for the development of thymic Foxp3+ CD4 regulatory T cells," Journal of Experimental Medicine, vol. 206, no. 13, pp. 3001-3014, 2009.

[48] M. Long, S. G. Park, I. Strickland, M. S. Hayden, and S. Ghosh, "Nuclear factor- $\kappa \mathrm{B}$ modulates regulatory $\mathrm{T}$ cell development by directly regulating expression of Foxp3 transcription factor," Immunity, vol. 31, no. 6, pp. 921-931, 2009.

[49] Q. Ruan, V. Kameswaran, Y. Tone et al., "Development of Foxp3(+) regulatory $t$ cells is driven by the c-Rel enhanceosome," Immunity, vol. 31, no. 6, pp. 932-940, 2009.

[50] Y. Zheng, S. Josefowicz, A. Chaudhry, X. P. Peng, K. Forbush, and A. Y. Rudensky, "Role of conserved non-coding DNA elements in the Foxp3 gene in regulatory T-cell fate," Nature, vol. 463, no. 7282, pp. 808-812, 2010.

[51] E. K. Deenick, A. R. Elford, M. Pellegrini, H. Hall, T. W. Mak, and P. S. Ohashi, "c-Rel but not NF- $\kappa$ B1 is important for T regulatory cell development," European Journal of Immunology, vol. 40, no. 3, pp. 677-681, 2010.

[52] G. Grigoriadis, A. Vasanthakumar, A. Banerjee et al., "C-rel controls multiple discrete steps in the thymic development of Foxp3+CD4 regulatory T cells," PLoS ONE, vol. 6, no. 10, Article ID e26851, 2011.

[53] A. J. Lee, X. Wu, H. Cheng, X. Zhou, X. Cheng, and S. C. Sun, "CARMA1 regulation of regulatory T cell development involves modulation of interleukin-2 receptor signaling," Journal of Biological Chemistry, vol. 285, no. 21, pp. 15696-15703, 2010.

[54] S. Rao, S. Gerondakis, D. Woltring, and M. F. Shannon, "cRel is required for chromatin remodeling across the IL-2 gene promoter," Journal of Immunology, vol. 170, no. 7, pp. 3724-3731, 2003.

[55] X. Tai, M. Cowan, L. Feigenbaum, and A. Singer, "CD28 costimulation of developing thymocytes induces Foxp3 expression and regulatory T cell differentiation independently of interleukin 2," Nature Immunology, vol. 6, no. 2, pp. 152-162, 2005.

[56] H. Bour-Jordan and J. A. Bluestone, "Regulating the regulators: costimulatory signals control the homeostasis and function of regulatory T cells," Immunological Reviews, vol. 229, no. 1, pp. 41-66, 2009.

[57] B. Salomon, D. J. Lenschow, L. Rhee et al., "B7/CD28 costimulation is essential for the homeostasis of the CD4+CD25+ immunoregulatory T cells that control autoimmune diabetes," Immunity, vol. 12, no. 4, pp. 431-440, 2000.

[58] Q. Tang, K. J. Henriksen, E. K. Boden et al., “Cutting edge: CD28 controls peripheral homeostasis of CD $4+\mathrm{CD} 25+$ regulatory $\mathrm{T}$ cells," Journal of Immunology, vol. 171, no. 7, pp. 3348-3352, 2003.

[59] L. H. Boise, A. J. Minn, P. J. Noel et al., "CD28 costimulation can promote $\mathrm{T}$ cell survival by enhancing the expression of $\mathrm{Bcl}-$ x(L)," Immunity, vol. 3, no. 1, pp. 87-98, 1995.
[60] J. S. Burr, N. D. L. Savage, G. E. Messah et al., "Cutting edge: distinct motifs within CD28 regulate $\mathrm{T}$ cell proliferation and induction of Bcl-XL," Journal of Immunology, vol. 166, no. 9, pp. 5331-5335, 2001.

[61] R. M. Thomas, L. Gao, and A. D. Wells, "Signals from CD28 induce stable epigenetic modification of the IL-2 promoter," Journal of Immunology, vol. 174, no. 8, pp. 4639-4646, 2005.

[62] C. Nazarov-Stoica, J. Surls, C. Bona, S. Casares, and T. D. Brumeanu, "CD28 signaling in T regulatory precursors requires p56lck and rafts integrity to stabilize the Foxp3 message," Journal of Immunology, vol. 182, no. 1, pp. 102-110, 2009.

[63] J. D. Fontenot, J. P. Rasmussen, M. A. Gavin, and A. Y. Rudensky, "A function for interleukin 2 in Foxp3-expressing regulatory $\mathrm{T}$ cells," Nature Immunology, vol. 6, no. 11, pp. 1142-1151, 2005.

[64] L. M. D'Cruz and L. Klein, "Development and function of agonist-induced CD25+Foxp3+ regulatory $\mathrm{T}$ cells in the absence of interleukin 2 signaling," Nature Immunology, vol. 6, no. 11, pp. 1152-1159, 2005.

[65] C. L. Bennett, J. Christie, F. Ramsdell et al., "The immune dysregulation, polyendocrinopathy, enteropathy, X-linked syndrome (IPEX) is caused by mutations of FOXP3," Nature Genetics, vol. 27, no. 1, pp. 20-21, 2001.

[66] B. R. Powell, N. R. M. Buist, and P. Stenzel, "An X-linked syndrome of diarrhea, polyendocrinopathy, and fatal infection in infancy," Journal of Pediatrics, vol. 100, no. 5, pp. 731-737, 1982.

[67] R. S. Wildin, F. Ramsdell, J. Peake et al., "X-linked neonatal diabetes mellitus, enteropathy and endocrinopathy syndrome is the human equivalent of mouse scurfy," Nature Genetics, vol. 27, no. 1, pp. 18-20, 2001.

[68] M. E. Brunkow, E. W. Jeffery, K. A. Hjerrild et al., "Disruption of a new forkhead/winged-helix protein, scurfin, results in the fatal lymphoproliferative disorder of the scurfy mouse," Nature Genetics, vol. 27, no. 1, pp. 68-73, 2001.

[69] V. L. Godfrey, J. E. Wilkinson, and L. B. Russell, "X-linked lymphoreticular disease in the scurfy (sf) mutant mouse," American Journal of Pathology, vol. 138, no. 6, pp. 1379-1387, 1991.

[70] H. P. Kim and W. J. Leonard, “CREB/ATF-dependent T cell receptor-induced FoxP3 gene expression: a role for DNA methylation," Journal of Experimental Medicine, vol. 204, no. 7, pp. 1543-1551, 2007.

[71] Z. Yao, Y. Kanno, M. Kerenyi et al., "Nonredundant roles for Stat $5 \mathrm{a} / \mathrm{b}$ in directly regulating Foxp," Blood, vol. 109, no. 10, pp. 4368-4375, 2007.

[72] E. Zorn, E. A. Nelson, M. Mohseni et al., "IL-2 regulates FOXP3 expression in human CD4+CD25+ regulatory T cells through a STAT-dependent mechanism and induces the expansion of these cells in vivo," Blood, vol. 108, no. 5, pp. 1571-1579, 2006.

[73] M. A. Burchill, J. Yang, C. Vogtenhuber, B. R. Blazar, and M. A. Farrar, "IL-2 receptor $\beta$-dependent STAT5 activation is required for the development of Foxp3+ regulatory T cells," Journal of Immunology, vol. 178, no. 1, pp. 280-290, 2007.

[74] G. Lal, N. Zhang, W. Van Der Touw et al., "Epigenetic regulation of Foxp3 expression in regulatory T cells by DNA methylation," Journal of Immunology, vol. 182, no. 1, pp. 259-273, 2009.

[75] S. Floess, J. Freyer, C. Siewert et al., "Epigenetic control of the foxp3 locus in regulatory T cells," PLoS Biology, vol. 5, no. 2, article e38, 2007.

[76] A. Toker and J. Huehn, "To be or not to be a Treg cell: lineage decisions controlled by epigenetic mechanisms," Science Signaling, vol. 4, no. 158, article pe4, 2011. 
[77] B. Liu, S. Tahk, K. M. Yee, G. Fan, and K. Shuai, “The ligase PIAS1 restricts natural regulatory $\mathrm{T}$ cell differentiation by epigenetic repression," Science, vol. 330, no. 6003, pp. 521-525, 2010.

[78] W. Ouyang, O. Beckett, Q. Ma, J. H. Paik, R. A. Depinho, and M. O. Li, "Foxo proteins cooperatively control the differentiation of Foxp3+ regulatory T cells," Nature Immunology, vol. 11, no. 7, pp. 618-627, 2010.

[79] L. F. Lu, T. H. Thai, D. P. Calado et al., "Foxp3-dependent microRNA155 confers competitive fitness to regulatory T cells by targeting SOCS1 protein," Immunity, vol. 30, no. 1, pp. 80-91, 2009.

[80] S. Kohlhaas, O. A. Garden, C. Scudamore, M. Turner, K. Okkenhaug, and E. Vigorito, "Cutting edge: the Foxp3 target miR-155 contributes to the development of regulatory T cells1," Journal of Immunology, vol. 182, no. 5, pp. 2578-2582, 2009. 


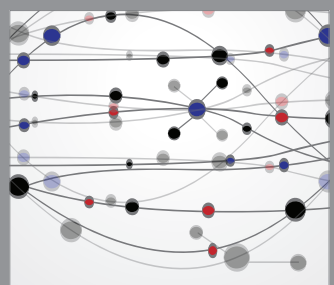

The Scientific World Journal
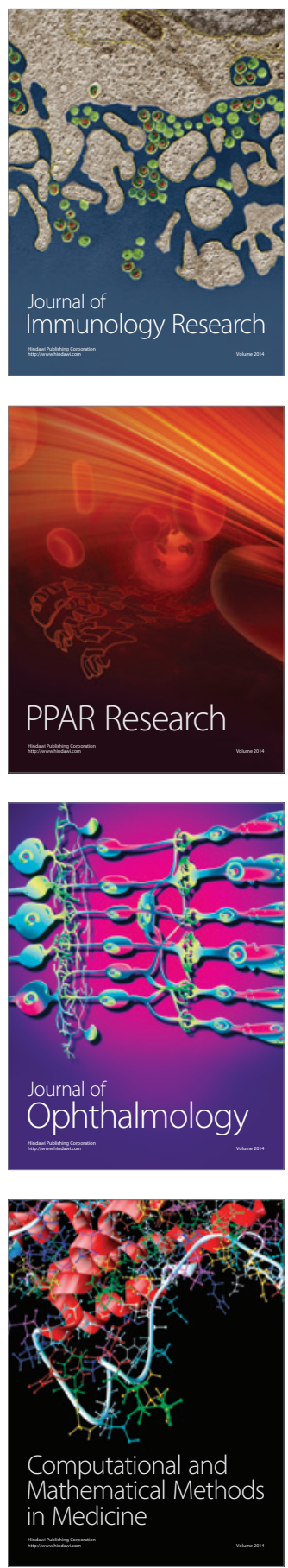

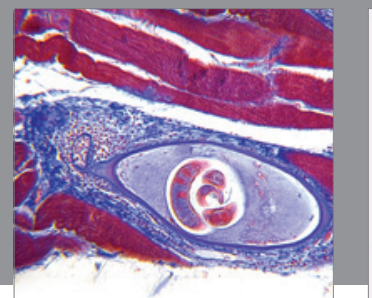

Gastroenterology

Research and Practice
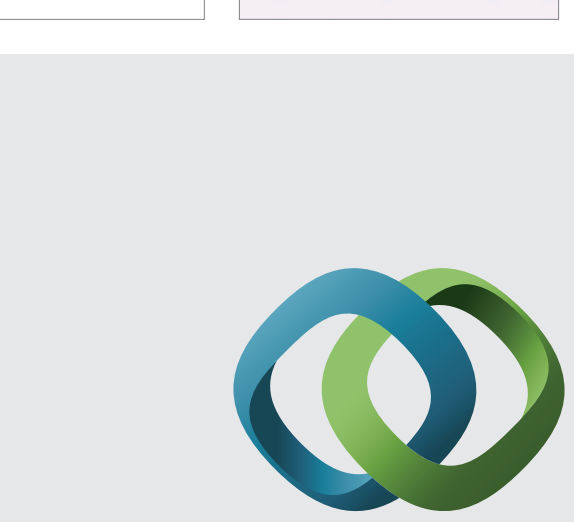

\section{Hindawi}

Submit your manuscripts at

http://www.hindawi.com
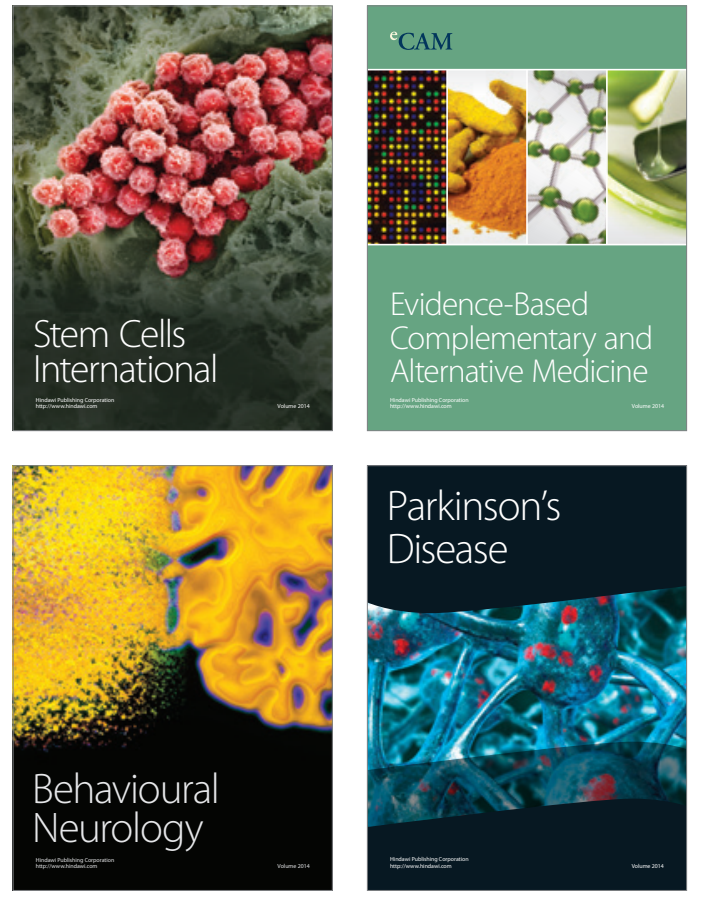
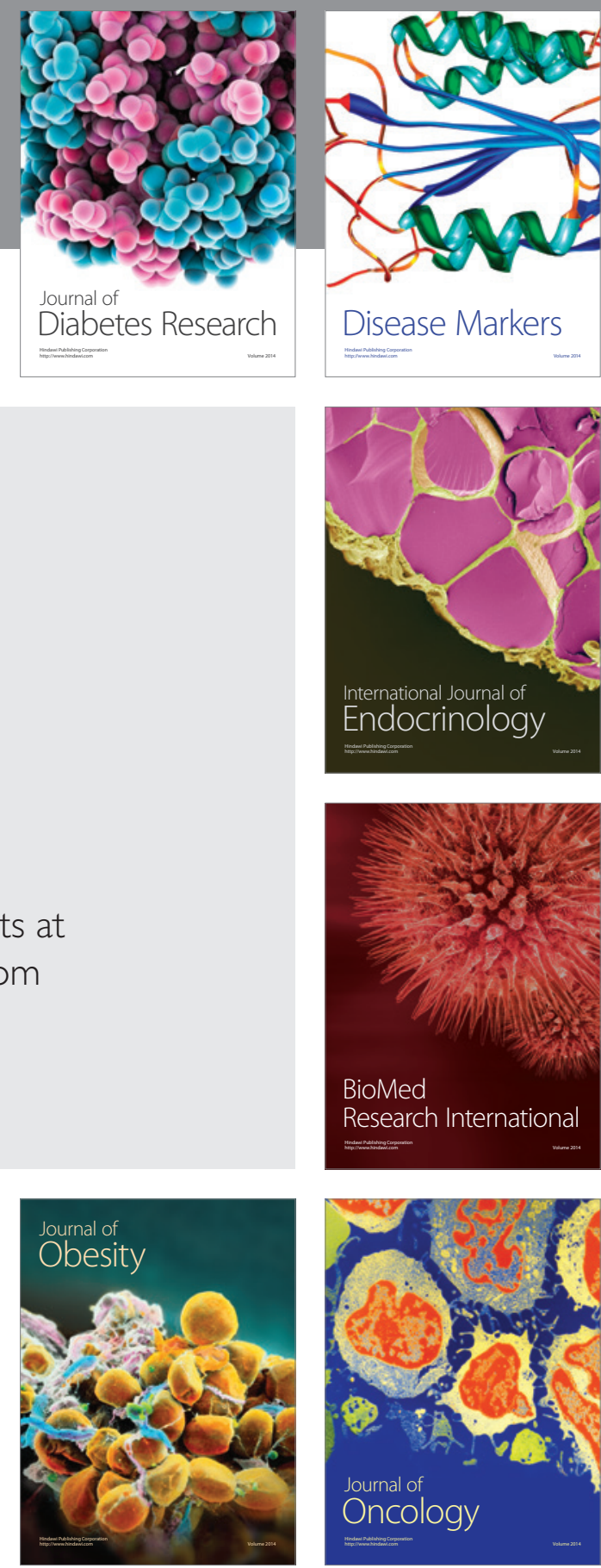

Disease Markers
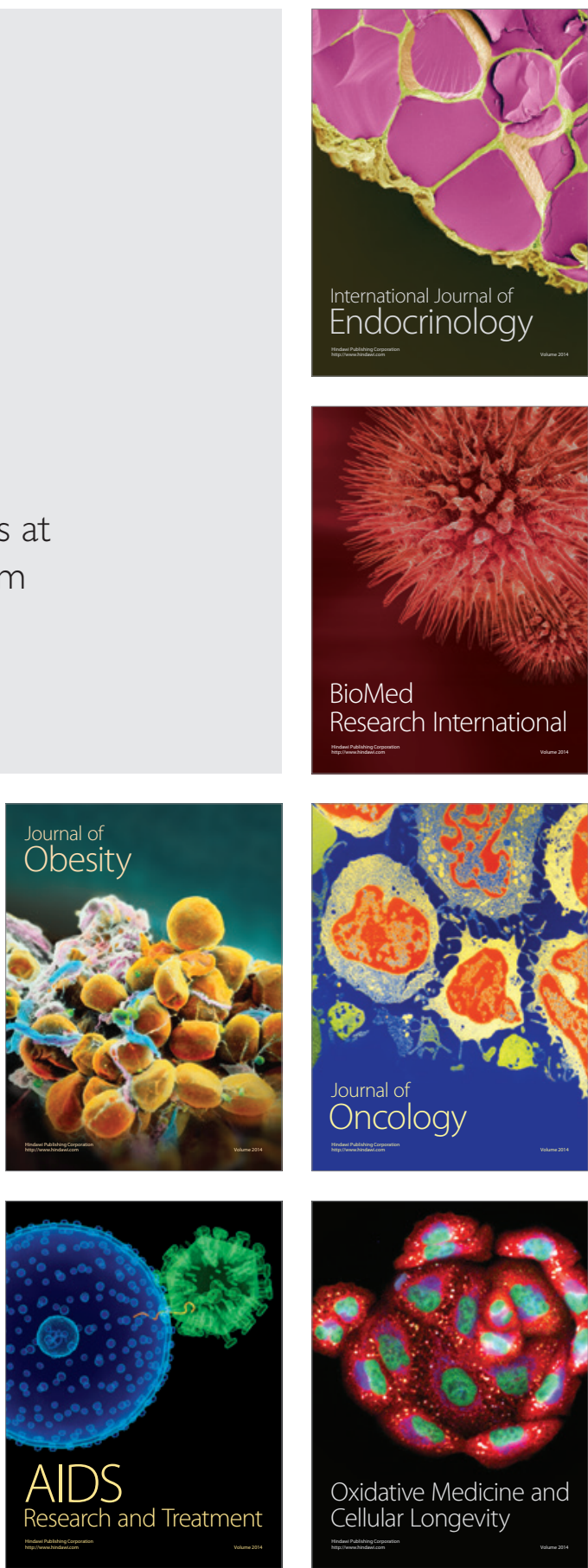